\title{
MYRA ORTH, Renaissance Manuscripts. The Sixteenth
}

\section{Century}

\section{Paola Cifarelli}

\section{(2) OpenEdition}

\section{Journals}

\section{Édition électronique}

URL : https://journals.openedition.org/studifrancesi/11687

DOI : $10.4000 /$ studifrancesi. 11687

ISSN : 2427-5856

\section{Éditeur}

Rosenberg \& Sellier

\section{Édition imprimée}

Date de publication : 1 avril 2018

Pagination : 119-120

ISSN : 0039-2944

\section{Référence électronique}

Paola Cifarelli, « MYRA orTh, Renaissance Manuscripts. The Sixteenth Century », Studi Francesi [En ligne], 184 (LXII | I) | 2018, mis en ligne le 02 juillet 2018, consulté le 15 novembre 2021. URL : http:// journals.openedition.org/studifrancesi/11687 ; DOI : https://doi.org/10.4000/studifrancesi.11687

Ce document a été généré automatiquement le 15 novembre 2021.

\section{(9) $\odot \Theta \Theta$}

Studi Francesi è distribuita con Licenza Creative Commons Attribuzione - Non commerciale - Non opere derivate 4.0 Internazionale. 


\title{
MYRA ORTH, Renaissance Manuscripts. The Sixteenth Century
}

\author{
Paola Cifarelli
}

\section{RÉFÉRENCE}

MYRA ORTH, Renaissance Manuscripts. The Sixteenth Century, London-Turnhout, Harvey

Miller Publishers, 2015, 2 voll., $339+382$ pp.

1 Nous tenons à rendre compte, même avec retard, d'un ouvrage qui constitue avant tout un hommage à la grande spécialiste en histoire de l'art et en codicologie qu'était Myra Orth, disparue prématurément en 2002, mais qui représente également une synthèse des recherches sur les manuscrits enluminés de la Renaissance, aussi bien du point de vue artistique que littéraire. Rédigé par l'auteur pendant de longues années et presque achevé au moment de sa mort, ce répertoire a été publié posthume, grâce à la collaboration entre l'éditeur, auprès duquel le tapuscrit avait été déposé, et un groupe de spécialistes qui ont été interpellés pour mettre à jour les références bibliographiques et les localisations, ainsi que pour combler les quelques lacunes mineures restées en suspens.

$2 \mathrm{Au} \mathrm{XVI} \mathrm{X}^{\mathrm{e}}$ siècle, le livre manuscrit continua à constituer un mode de circulation des textes littéraires pratiqué régulièrement, bien que concurrencé par les imprimés. Utilisé pour une diffusion officielle ou intime des textes, le manuscrit a pu constituer un objet d'art raffiné, original et d'une grande qualité artistique. Un répertoire qui recense les cent codex les plus représentatifs des goûts et des tendances des élites à la Renaissance ne peut donc que rendre les plus grands services à la communauté scientifique et aux littéraires seiziémistes. La tranche temporelle retenue (1515-1570) se justifie pleinement par le cours des évènements historiques, qui influencèrent considérablement le développement culturel et artistique de la France au XVI ${ }^{\mathrm{e}}$ siècle.

Le premier volume débute par une Introduction (pp. 25-47) consacrée à l'évocation des éléments du cadre politique littéraire et artistique susceptibles d'avoir influencé la 
production des manuscrits artistiques, avec une attention particulière pour le développement des traductions, le poids de la Réforme et de la Contre-Réforme, l'empreinte de l'art italien; les relations complexes qui se tissent entre manuscrit et imprimé à l'époque concernée font l'objet d'un paragraphe à part, dans lequel les manuscrits sont envisagés non seulement en tant qu'objets matériels destinés à la diffusion de la culture, mais aussi comme enjeux commerciaux. Un paragraphe consacré à l'identité artistique des livres manuscrits permet d'évoquer les lieux de production, les groupes d'enlumineurs et leurs rapports avec les libraires, les caractéristiques des différents éléments décoratifs. Ce premier chapitre se clôt sur un survol des expositions consacrées aux manuscrits enluminés de la Renaissance aux XIX et $\mathrm{xx}^{\mathrm{e}}$ siècles. Deux autres chapitres dans le premier volume traitent respectivement des styles caractérisant les bordures, importantes pour situer les codex dans leur contexte artistique, et de la production de l'anversois Noël Bellemare, peintre et dessinateur actif à Paris dans la première moitié $d u \mathrm{XvI}^{\mathrm{e}}$ siècle qui imposa son propre style aux producteurs de manuscrits parisiens, particulièrement dans le domaine des livres d'heure.

4174 splendides illustrations, en couleur et en noir et blanc, constituent la partie la plus importante de ce premier tome; la qualité remarquable de ces reproductions, leur dimension et la présence d'images de comparaison, permettant de mettre l'iconographie des manuscrits en parallèle avec d'autres ouvrages d'art, rendent très utile cette section non seulement pour les historiens de l'art. Enfin, une biographie des artistes et des scribes connus ayant travaillé aux manuscrits recensés, ainsi que des auteurs et translateurs des ouvrages transcrits dans les codex complètent les informations contenues dans le deuxième tome. Celui-ci se compose de cent fiches, consacrées à chacun des manuscrits retenus dans le catalogue. Dans les articles, classés d'après la cote du manuscrit, on trouvera la mention de l'ouvrage copié dans le codex, les données codicologiques essentielles (format, constitution des cahiers, reliure) ainsi que le relevé des miniatures, la description de la décoration et les informations concernant le type d'écriture utilisée, très utiles pour situer le codex dans son milieu culturel. Ensuite, une analyse du texte copié et des données iconographiques permet de réunir et de synthétiser une masse de données souvent dispersées; les historiens de l'art seront donc à même d'avoir aussi un premier contact avec les études littéraires consacrées au texte copié dans le codex, tandis que les spécialistes en littérature de la Renaissance pourront trouver les informations iconographiques et artistiques concernant le manuscrit, indispensables pour compléter l'étude littéraire et philologique. Une grande partie des volumes répertoriés dans ce catalogue a fait l'objet d'études approfondies de la part de M. Orth elle-même, ce qui rend les notices particulièrement utiles surtout pour les notions concernant l'histoire de l'art; en effet, l'A. a consacré de nombreux travaux à l'identification de la main des différents enlumineurs, au contexte auquel ceux-ci se rattachent, aux rapports entre les différents milieux artistiques et les commanditaires, en contribuant à tracer un tableau précis de l'ornementation du livre à la Renaissance. Les historiens de la littérature trouveront donc dans ce répertoire une mine d'informations, mais aussi des indications bibliographiques précieuses. En effet, chaque notice se clôt avec les indications concernant les expositions dans lesquelles chaque codex a été exposé, les indications sur les anciens propriétaires mais surtout la liste des études consacrées à la décoration et aux textes. De nombreux index (de l'iconographie, des types d'ouvrages copiés, des provenances, des manuscrits eux-mêmes, des illustrations) complètent le répertoire. 
5 Comme il est inévitable dans ce genre d'ouvrages, des imprécisions ou des lacunes se glissent parfois dans les notices pour les notions concernant les aspects littéraires ou philologiques; il n'en reste pas moins que ce répertoire constitue un instrument de travail précieux pour tous les seiziémistes qui s'intéressent au livre manuscrit illustré. 